\title{
An Empirical Analysis of Volatility Effect of International Financial Market based on MSV Model
}

\author{
Xuexue Tang \\ Department of Educational Administration Management, \\ Xi'an Peihua University, Xi'an, China \\ 562671195@qq.com
}

Keywords: International financial markets; Volatility benefit; Stochastic volatility MSV model

\begin{abstract}
The fluctuations in the International Financial Markets (IFM) are mutually reinforcing, and they truly show the characteristics of the fluctuations of the IFM. Previously, theories and methods of general economic contrasting construction are based on static floating effects, and there is no way to fully and truly reflect the objectively fluctuating effects among IFM. In 1982, the famous American economist Robert pioneered the ARCH theory of the heteroscedasticity of the auto-regression conditions for the first time. Since then, there have been rapid changes in the scope of the theory of volatility between IFM. More and more financial market fields have gradually evolved from their configurations.
\end{abstract}

\section{Literature Review}

This paper describes the volatility spillover effect, which means that the instability created in the IFM is not only influenced by its own floating components, but also affected by the volatility of financial markets from other regions. It is the causal relationship between the second-order matrix of the rate of return. We conduct research and analysis based on the direction of volatility spillovers between IFM, and recognize the ways in which the volatility spreads and the direction of dissemination. Therefore, to a large extent, we can understand and grasp the method of dissemination of information among IFM.

\subsection{Foreign Status}

In the research on the volatility spillover effect between IFM, the most important economists in various countries are the use of vector autoregressive theory VAR, co-integration analysis, GARCH theory and stochastic volatility MSV theory. Among them, the most basic configuration used is the MSV model. Foreign economists mostly applied this model to the study of the mutual influence among the stock markets. The earliest method was the American economist Kanas. Kanas [1] used the MSV model to analyze the stock markets of Europe and the United States and six countries. The correlation of the market was analyzed and the results of the analysis proved that the stock market has a volatility spillover effect. The well-known economists Bodart and Reding [2] used binary MSV model data to conduct in-depth research on the relationship between exchange rate fluctuations and the returns of the five European countries' seven industry stock markets. Their conclusions also prove that the fluctuations with the obvious form of the spillover effect. Caporale also used the SV model structure to explore the volatility spillover effect between the stock prices of East Asian countries and exchange rate returns [3]. We can see from this that there is not only a single stock exchange to the foreign exchange market. The spillover effect, at the same time, there is another two-way volatility spillover effect in the stock markets of Indonesia and Thailand. The well-known economist Walid are also studying MS-MSV theoretical models established when the foreign exchange rate changes in the four emerging regions of Hong Kong, Malaysia, Mexico and Singapore affect the stock market, and found that the volatility spillovers in foreign exchange markets and stock markets. The different states exhibited during the period of "smoothness" and "unsettlement" indicates that the instability of the international currency exchange rate will simultaneously cause fluctuations in the stock indexes of the international stock market.

\subsection{Domestic Status}

At present, China's economists have gradually increased the use of stochastic volatility MSV configuration to study the foreign exchange market and its volatility spillover relationship with the stock market. The economists Ba Shusong and Yan Min [4] used multivariate MSV models and daily statistical 
data to demonstrate and interpret the dynamic relationship of China's stock prices. They found that only the foreign exchange market exists in terms of volatility spillover effects. The short-term one-way guidance effect between stock markets has asymmetrical volatility spillover effect on the foreign exchange market in the financial market's floating overflow. However, there is a symmetrical volatility spillover effect on the stock market in the foreign exchange market. China did not reflect the long-term equilibrium symbiosis between stock prices and exchange rates. Li Cheng [5] is also forming a comprehensive analysis of the volatility spillover effect of China's major financial markets through the establishment of MSV configuration. The research results show that there is a clear two-way volatility spillover effect between the stock market and the foreign exchange market. This two-way volatility spillover effect is most likely due to the mutual influence of markets. The economists Zhu Xinling and Li Peng [6] used Greenberg's causality test, BEEK model, and MSV model, and conducted in-depth research into the stock price from three perspectives: the local average overflow, volatility spillover, and dynamic correlation. The volatility spillover effect shows that there is a two-way spillover effect and dynamic correlation between the foreign exchange market of China and the stock market mayor, and there is some "asymmetry", which means that the fluctuation of the exchange rate has a less than the Shanghai Securities Index fluctuations have a significant impact on the exchange rate. Su Chengjian [7] used MSV analysis to analyze the signals of the overall index of the Shanghai Stock Exchange and the Shenzhen Stock Exchange Index, that is, split into high-frequency signals and low-frequency signals, and then used regression analysis to compare the Shanghai and Shenzhen stocks. The correlation between high-frequency signals in the stock market and the conclusion analysis shows that there is a clear spillover effect between Shanghai and Shenzhen stock markets.

In general, the empirical research on the spillover effects of MSV-type construction on China's stock market and foreign exchange is inconsistent, and most of them only seek exploration and argument from the perspective of time. Therefore, the study of the spillover effect between the mainland and foreign stock markets listed in this article will use wavelet multi-resolution analysis and multi-MSV configuration to link the observation of international stock markets from two different dimensions of time and frequency.

\section{Research Ideas and Methods}

\subsection{Theoretical Analysis of Volatility Spillover Effect}

The theory of multivariate floating rate configuration has extremely important application value and broad prospects in the areas of optimal investment portfolio, crisis management, and asset allocation. It can provide extremely important arguments for the decision makers in the financial sector. Moreover, because financial floats conduct their own transmissions between different resources and financial markets, modeling of fluctuation effects under a diversified theoretical framework can significantly increase the statistical application of industry data. Corresponding to the ARCH configuration is the stochastic volatility SV configuration proposed by the economist Tayor. Tayor thinks that the sequence heterogeneity is determined at the same time as the random variable produced in its potentially random process. In the $\mathrm{ARCH}$ configuration, spikes and valleys and the lasting memory of the income squared sequence are two extremely typical characteristics of the international financial time series. It shows more obvious advantages in China's financial market. Yu Shouhong's research further proves the SV. Compared with the ARCH configuration, the configuration has a strong depiction of financial data. Therefore, this paper adopts the SV configuration and introduces the MSV configuration to discuss the floating overflow between China's stock market. Combined with the general verification and inspection of computer software, the obtained configuration data is compared with other data estimation methods.

\subsection{Data Selection}

As the global stock market continues to suffer from the turmoil of various global crises such as the financial crisis and the European debt crisis, the general plan for this paper will be closer to the Shanghai 
Composite Index from August 29, 2009 to August 28, 2012. The Hong Kong Hang Seng Index, the Standard Poole 500 Index and the Nikkei 225 Index's daily closing price data, and then using the MSV configuration to explore the floating spillover mechanism between them will allow us to further control our country. When the international stock market is frequently fluctuating, we can use the information transmission direction to plan the adjustment of investment strategy and position in real time.

The comprehensive framework data selected in this paper can be used as the representatives of the stock markets of mainland China, Hong Kong, the United States, and Japan in succession. Several daily closing prices representing the stock market are extracted as the original experimental data for our exploration to understand the floating spillover effect between them. All experimental data in this article comes from Yahoo Finance. Given the difference in stock index bases, we replaced the stock index with:

$$
R_{i, t}=\left(\operatorname{In} P_{i, t}-\operatorname{In} P_{i, t-1}\right) \times 100, \mathrm{i}=1,2,3,4 .
$$

Where $P_{i}, t$ represents the closing price of the $t$-th trading day in the $i$-th market, and $R_{i}, t$ represent the yield of the $i$-th market.

Due to the inconsistency of the buying and selling data caused by the inconsistency of time between different regions and the inconsistency of holidays, the initial experimental data was pre-processed, applying the US stock market $t-1$ trading day data and China's, Hong Kong and Japan's stock market $t$. The data for each trading day correspond, and the out-of-sync data caused by festivals in each country is eliminated. Finally, we obtained 709 samples of the S\&P 500 index, 692 of the Nikkei 225 index, and 724 samples of the Hang Seng index.

\subsection{Statistical Description}

The methods used for stochastic fluctuations in the SV configuration include various ways of estimating the general regression configuration. For stochastic volatility SV configuration, although its configuration is relatively intuitive, and can be well combined with actual data, there is a great deal of difficulty in the parameter estimation of this configuration. At present, the methods adopted by international economic academia to estimate the parameters of SV configuration can be roughly divided into two categories: one is to try to obtain the full likelihood function form, such as simulation; the other is to rely on certain Some formula theorems have obtained likelihood functions, such as the generalized moment method. However, this type of method is not very effective compared to the first type.

Table I. Basic statistical characteristics of stock returns

\begin{tabular}{|c|c|c|c|c|}
\hline & $\mathrm{R} 1, \mathrm{t}$ & $\mathrm{R} 2, \mathrm{t}$ & $\mathrm{R} 3, \mathrm{t}$ & $\mathrm{R} 4, \mathrm{t}$ \\
\hline Mean & -0.0055 & 0.0063 & -0.0012 & -0.0022 \\
\hline Maximum & 0.5845 & 0.6571 & 0.557551 & 0.6073 \\
\hline Min & -0.6603 & -0.9771 & -0.588659 & -1.2241 \\
\hline $\begin{array}{c}\text { Standard } \\
\text { deviation }\end{array}$ & 0.1643 & 0.1689 & 0.1330 & 0.14611 \\
\hline Skewness & -0.3311 & -0.3990 & -0.3215 & -0.8990 \\
\hline Kurtosis & 4.2940 & 6.6274 & 4.7159 & 10.5669 \\
\hline Jarque-Bera & 62.4209 & 406.9622 & 99.0583 & 1744.453 \\
& $(0.0000)$ & $(0.0000)$ & $(0.0000)$ & $(0.0000)$ \\
\hline Q(10) & 10.5543 & 9.4383 & 17.4883 & 24.6731 \\
& $(0.2611)$ & $(0.3100)$ & $(0.0023)$ & $(0.0002)$ \\
\hline Q2(10) & 140.0019 & 106.6729 & 251.2055 & 187.0219 \\
& $(0.0000)$ & $(0.0000)$ & $(0.0000)$ & $(0.0000)$ \\
\hline ADF & -50.29 & -47.99 & -51.01 & -47.31 \\
& $(0.0000)$ & $(0.0000)$ & $(0.0000)$ & $(0.0000)$ \\
\hline PP & -51.13 & -47.98 & -52.66 & -47.49 \\
& $(0.0000)$ & $(0.0000)$ & $(0.0000)$ & $(0.0000)$ \\
\hline
\end{tabular}

Table I shows the general descriptive statistical characteristics of the series of returns $R i$, $t$ for each stock index. From the standpoint of standard deviation and maximum value, the S \& P 500 index has a relatively 
large standard deviation and maximum value, indicating that its advantages are very obvious. $\mathbf{R}_{1, t}, \mathrm{R}_{2, t}, \mathrm{R}_{3, t}$, $\mathrm{R}_{4, t}$ in Table I represent the returns of the Shanghai Composite Index, the Standard \& Poor's Index, the Hong Kong Hang Seng Index and the Nikkei Index, respectively.

\section{Empirical Analysis Results}

\subsection{Model Parameter Estimation Results}

According to the MSV configuration, 50,000 calculations are performed on the parameters given, and the first 4000 operations are eliminated as pre-sale data, 4001 to 50000 operational data samples are saved, and finally we obtain the necessary parameter estimates such as shown in table 2 . Here, it is assumed that the parameters are separated from each other and the prior dispersion is set to:

$$
\begin{gathered}
U 1 \sim N(0,25), U 2 \sim N(0.25), \mathrm{P}_{\varepsilon} \sim U(-1,1) \\
\varphi_{11}^{*} \sim \operatorname{Beta}(20,1.5), \varphi_{22}^{*} \sim \operatorname{Beta}(20,1.5), \\
\varphi_{12} \sim N(0,10), \varphi_{21} \sim N(0,10), \\
\sigma_{\eta 1}^{2} \sim I G(2.5,0.025), \sigma_{\eta 2}^{2} \sim I G(2.5,0.025)
\end{gathered}
$$

Where, $\varphi_{11}^{*}=\left(\varphi_{11}+1\right) / 2 ; \varphi_{22}^{*}=\left(\varphi_{22}+1\right) / 2 ; I G$ represent the inverse Gamma distribution.

Table II shows the mean, standard deviation, and MC errors for MSV configuration parameter estimates. Due to the limitations of space, the quantile values of the estimated values of various parameters are not given in the table. From Table II, it can be found that the standard deviation and MC gap of each data are very small, and the MC error is smaller than the standard deviation. A relatively small MC error means that the configuration has higher accuracy and is far from the real dispersion. The deviation is smaller, indicating that the sampling has converged, and the parameter estimates of the configuration are relatively accurate.

Table II. Parameter Estimation Results for MSV Models by Each Index

\begin{tabular}{|c|c|c|c|c|c|c|c|c|c|c|}
\hline Index & index & $\mathrm{U} 1$ & $\mathrm{U} 2$ & $\varphi_{11}$ & $\varphi_{12}$ & $\varphi_{22}$ & $\varphi_{21}$ & $\mathrm{P} 1$ & $\sigma_{\eta 1}$ & $\sigma_{\eta 2}$ \\
\hline \multirow{3}{*}{$\mathrm{S} \& \mathrm{P}$} & Mean & -3.75 & -3.78 & 0.769 & 0.042 & 0.946 & 0.426 & 0.259 & 0.132 & 0.152 \\
\cline { 2 - 11 } & $\begin{array}{c}\text { Standard } \\
\text { deviation }\end{array}$ & 0.107 & 0.304 & 0.146 & 0.019 & 0.043 & 0.373 & 0.047 & 0.069 & 0.060 \\
\cline { 2 - 11 } & MC error & 0.006 & 0.014 & 0.009 & 0.001 & 0.003 & 0.024 & 0.001 & 0.005 & 0.004 \\
\hline \multirow{3}{*}{ Nikkei } & Mean & -3.79 & -4.14 & 0.920 & -0.03 & 0.894 & 0.031 & 0.367 & 0.142 & 0.235 \\
\cline { 2 - 11 } & standard value & 0.160 & 0.148 & 0.063 & 0.027 & 0.055 & 0.028 & 0.041 & 0.059 & 0.064 \\
\cline { 2 - 11 } & MC error & 0.008 & 0.005 & 0.004 & 0.002 & 0.003 & 0.004 & 0.039 & 0.004 & 0.004 \\
\hline \multirow{2}{*}{$\begin{array}{c}\text { Hang } \\
\text { Seng }\end{array}$} & Mean & -3.75 & -4.17 & 0.785 & 0.173 & 0.968 & 0.454 & 0.604 & 0.169 & 0.106 \\
\cline { 2 - 11 } & standard value & 0.310 & 0.363 & 0.147 & 0.044 & 0.027 & 0.182 & 0.070 & 0.093 & 0.023 \\
\cline { 2 - 10 } & MC error & 0.021 & 0.022 & 0.009 & 0.002 & 0.002 & 0.012 & 0.005 & 0.006 & 0.001 \\
\hline
\end{tabular}

\subsection{Wave effect check}

The verification of the floating spillover effect between stock markets in Shanghai, the United States, Japan, and Hong Kong can be viewed from the volatility continuity system $\varphi 11, \varphi 22$, which represents the influence of the early fluctuations of each group of independent index financial markets. The values of $\varphi 11$ and $\varphi 22$ are all very significant. The closeness to 1 indicates that the floating of the four stock markets in the future will be affected by their own up-front fluctuations, and the continuity of fluctuations will be strong, and the volatility clustering will be very obvious.

Since the 1990s, the process of global integration within the economic sphere has gradually intensified. This has also led to a deepening of the opening level of financial markets in all countries, and a large amount of capital is rapidly and massively free on a global scale. The process of economic global integration has greatly enhanced the interrelationship between IFM. It is this interrelationship between global financial markets that makes it possible that the volatility of the financial markets of any country in any region can rapidly spread to other regions. The country's financial markets may thus lead to a wide 
range of global financial crises. Therefore, studying the law of the volatility spillover effect between IFM is very important and far-reaching significance for effectively avoiding the global financial risks. At the same time, due to the existence of this kind of floating sustainability, the same link exists between the floating spillover effect between markets and the associated floating continuities associated with them.

\section{Summary}

In the current global economic globalization process, how to effectively avoid, prevent and monitor the risks of financial markets has attracted the attention of all countries. We have systematically developed the floating modeling theory that has prevailed in economic and academic circles in the past two decades. In summary, the two major main configurations of the floating effect are also interpreted: the modeling characteristics, parameter estimation, and model checking of the ARCH configuration and stochastic fluctuating SV-configuration. The continuity of the floating effect is currently prevailing internationally. The main point of view is that it is based on the root-meeting phenomenon of the floating process or depicted by the divisional behavior of the fluctuating process. We extend the determination of the sustainability of the floating effect of the IFM from the implicit factorization process to the unit root of the characteristic equations. The fluctuations in the continuity of the floats are dependent on each other, and they are typically correlated. Perspectives on the relevant conditions in the study illustrate the relationship between volatility continuity and the typical correlation of sequences. The theory of fluctuation effects still faces many difficulties. Parameter estimation and model checking has become a major obstacle to our progress. The effective estimation method still needs to be proposed; the persistence of volatility still lacks a reasonable and consistent definition; the relationship between the two types of volatility models remains It is still not clear; the precise description of the characteristics of peak heavy tails is still doubtful. In short, there are still many problems that need to be further studied.

Judging from the effectiveness of the demonstration, the risk of floating stocks in the external stock markets of the Shanghai stock market is mostly concentrated in the Hong Kong stock market as well as in the US stock market. Therefore, the control of the external floating dangers in the Shanghai stock market not only requires us to pay attention to the Hong Kong stock market in the short term, and also to pay attention to the trend of the US stock market in a timely manner. The value fluctuation of the US stock market will directly affect the market confidence of Chinese investors, which in turn will have a fluctuating effect on China's stock market. The level of openness between the mainland stock market and the Hong Kong stock market is relatively high. In particular, many large mainland companies have preferred to list in Hong Kong in Hong Kong over the years, and then return to the Mainland for cross-listing activities. This has increased the connection between the mainland and Hong Kong stock market. The floating value of the Hong Kong stock market will directly affect the stock price of the mainland stock market. The fluctuation of the stock market in our country will certainly be affected by the fluctuation of other financial markets, but if the degree of verification is slightly larger, its level is not obvious. This shows that China's capital market has not yet fully opened, cross-border capital flows have been strictly controlled, and capital management can also resist other stock market crises to a large extent. However, from a long-term point of view, the Chinese stock market will gradually open up to the outside world, and foreign resources will enter the Shanghai and Shenzhen stock markets more. This will gradually strengthen the link between the Chinese stock market and other stock markets, and the floating spillover effect will also increase.

Project Fund: Research on the dynamic mechanism of RMB regional internationalization under the "One Belt and One Road" strategy, Xi'an Social Science Planning Fund Project (2016). Project No.: 16PH04; Project Rating: Key Projects; Project Host: Tang Xuexue 


\section{References}

[1] Kanas A. Volatilitys pillovers between stock returns and exchange rate changes international evidence [J]. Journal of business finace \& accounting 2000, 447-476.

[2] Masry Eias, Fag Tjostheim. Non parametric estimation and identification of nonlinear ARCH time series - strong convergence and asymptotic normality, Econometric Theory, 1995. 11. 258-289.

[3] Xiong Zhengde, Xie Min. Theoretical Research and Evaluation of Volatility Spillover Effects among Financial Markets, Journal of Productivity Research, 2008, (1):51-53.

[4] Ba Shusong, Yan Min. The dynamic relationship between stock prices and exchange rates-based on the empirical analysis of the Chinese market, Journal of Nankai Economic Research, 2009, (2):46-60.

[5] Li Cheng, Ma Wentao, Wang Bin. Research on the Spillover Effect among China's Financial Market-Based on the Analysis of the Quaternary VAR-GARCH(1,1)-BEKK Model, Journal of Quantitative Economic and Technological Economics Research, 2010, (6): 3-18.

[6] Zhu Xinling, Li Peng. The linkage effect of RMB exchange rate and stock price -- Based on spillover and dynamic correlation perspective, Journal of Financial Theory and Practice, 2011, (5): 8-12.

[7] Su Chengjian, Liu Xing, Liu Lipei, et al. Application of Wavelet Analysis Method to Study the Spillover Effects of Shanghai and Shenzhen Stock Markets, Journal of Systems Engineering, 2004, 19(1): 99-103. 